\title{
Minimalism, Supervaluations and Fixed Points
}

\author{
Sergi Oms, University of Barcelona, Logos Group
}

\begin{abstract}
In this paper I introduce Horwich's deflationary theory of truth, called 'Minimalism', and I present his proposal of how to cope with the Liar Paradox. The proposal proceeds by restricting the T-schema and, as a consequence of that, it needs a constructive specification of which instances of the T-schema are to be excluded from Minimalism. Horwich has presented, in an informal way, one construction that specifies the Minimalist theory. The main aim of the paper is to present and scrutinize some formal versions of Horwich's construction.
\end{abstract}

Keywords: Minimalism, deflationism, Paul Horwich, Saul Kripke, fixed points constructions

Paul Horwich is a deflationist about truth. This means that, according to him, truth is not a genuine or robust property, but a deflationary one, in the sense that, properly speaking, the truth predicate is not used to describe anything: truths do not share any interesting common property. In general, deflationists think that asserting that something is true is equivalent to asserting that very something itself; although the nature of that equivalence may vary from one philosopher to another, of course. Moreover, deflationists defend that nothing more is needed beyond this equivalence to explain all facts concerning truth. The equivalence between sentences and their truth ascriptions is usually captured by deflationists via the T-schema, which in the case of Horwich, is applied to propositions:

$$
\text { (T-schema) }<p>\text { is true iff } p .^{1}
$$

Horwich has presented and defended his theory of truth in a number of places (see, especially, Horwich 1998, 2001, 2010b). That theory, which is

\footnotetext{
${ }^{1}$ The symbols ' $<$ ' and ' $>$ ' surrounding a given expression $e$ produce an expression referring to the propositional constituent expressed by $e$. Thus, when $e$ is a sentence, ' $\langle e\rangle$ ' means the proposition that $e$.
} 
called 'the Minimalist theory of truth' or 'Minimalism', contains as axioms all instances of the T-schema applied to propositions, and nothing else. ${ }^{2}$

One of the main theses of Minimalism is that the instances of the Tschema are conceptually, explanatorily and epistemologically fundamental. In the first place, they are conceptually fundamental because the basic and fundamental regularity of use that determines the meaning of 'true' (which is, according to Horwich, the concept of truth) is our disposition to accept all instances of the T-schema (Horwich 1998, p. 145). In the second place, they are explanatorily fundamental because the instances of the T-schema are all we need to explain all our uses of 'true'. ${ }^{3}$ Finally, they are epistemologically fundamental due to the fact that the instances of the T-schema are "immediately known"(Horwich 2010b, page 36): they cannot be deduced from anything more basic.

As it is well known, however, the proposition that asserts its own untruth (let us call it 'the Liar') makes the theory consisting solely of all instances of the T-schema inconsistent in classical logic (the argument that proves such inconsistency is the Liar Paradox). ${ }^{4}$ Until recently, Horwich's response to this problem was very succinct. In his (1998) he claims that the lesson the Liar teaches us is that not all the instances of the T-schema are to be included as axioms in the theory of truth (Horwich 1998, page 42). Consequently, the Minimalist theory must consist of a restricted collection of instances of the T-schema: only those that do not engender Liar-like paradoxes. Precisely which of the instances of the T-schema should be removed was, nonetheless, left undetermined.

In this paper, I want to explore some constructive specifications, based on Horwich (2010a), in order to specify which instances of the T-schema should be excluded from the Minimalist theory of truth. After briefly introducing Horwich's stance on the Liar Paradox in the next section, such constructions will be presented and explored in the following four sections. Finally, in section 6, I will offer some discussion regarding them.

\footnotetext{
${ }^{2}$ This characterization is not completely accurate. As Horwich admits, the theory should also have an axiom claiming that only propositions are bearers of truth (see Horwich 1998, fn. 7 on page 23, page 43). Moreover, as we are going to see, some of the instances of the T-schema will not be included in the theory, due to the paradoxes of truth.

${ }^{3}$ This is an exaggeration; strictly speaking, we will need other theories besides the theory of truth to explain all the facts about truth, because some of those facts will involve other phenomena. As Horwich says, Minimalism "provides a theory of truth that is a theory of nothing else, but which is sufficient, in combination with theories of other phenomena, to explain all the facts about truth" (Horwich 1998, pp. 24-25). See also the discussion below.

${ }^{4}$ I assume that the reader is familiar with the Liar Paradox.
} 


\section{Truth and Vagueness}

A proposal for a solution of the Liar Paradox within Horwich's framework has been made explicit by Armour-Garb (2004), Beall and ArmourGarb (2005), Restall (2005) and, though succinctly, by Horwich himself in his (2010) (Beall, Armour-Garb and Restall have called it 'Semantic Epistemicism'). The Minimalist stance with respect to The Liar consists of the following two tenets:

1. The Liar is true or the Liar is false.

2. It is conceptually impossible to know whether the Liar is true or false.

Let us briefly consider the rationales for 1 and for 2 . First, 1 is an instance of the principle of bivalence, which, according to Horwich (1998, page 71), follows from the law of excluded middle (LEM), which, in turn, is seen by him as a basic law of thought. ${ }^{5}$

The reasons for accepting 2, claims Horwich, are the same as in the case of vagueness (Horwich 2010b, fn. 11 on page 91). Horwich defends an epistemic account of vagueness according to which vague predicates have sharp boundaries (so that LEM is preserved) which we are not capable of knowing. This is so because the only way we can be justified in applying a given vague predicate to an object is via the fundamental facts underlying the vague predicate. Moreover, according to Horwich, those fundamental facts are certain partial functions which specify the subjective probability of the vague predicate in question being applied to a certain object. The fact that these functions remain silent with respect to the application of the predicates to certain objects explains why we cannot know whether those objects are within the extensions of vague predicates. ${ }^{6}$

As I said, Horwich's strategy for dealing with the Liar Paradox consists of applying his view of vagueness to the case of the truth predicate. So, the reasons for accepting tenet 2 are rooted in the fact that the instances of the T-schema in the Minimalist theory of truth are explanatorily fundamental with respect to the truth predicate in the same sense as the partial functions

\footnotetext{
${ }^{5}$ Whether bivalence is available to Horwich is a highly contentious matter. The fact that Minimalism does not contain the instance of the T-schema for the Liar sentence seems to be enough to block the derivation of this instance of bivalence from LEM (see, especially, Beall and Armour-Garb 2005, sec. 5.2 and Schindler 2018, sec. 4). For the sake of argument, in this paper I will suppose that Horwich can overcome this difficulty. One way to do that might involve understanding falsity as mere untruth. I will not pursue this issue further in this paper though. Thanks to two anonymous referees for prompting this clarification.

${ }^{6}$ For further details see Horwich $(1997,2005)$.
} 
mentioned in the case of vagueness are explanatorily fundamental with respect to the vague predicates. In order to know the truth value of the Liar, we would need to be justified in ascribing the truth value in question to the Liar; and such justification can only stem from the Liar instance of the T-schema. However, since that instance is not within the Minimalist theory, we cannot know its truth value. The impossibility of knowing the truth value of the Liar is caused, according to Horwich, by the meaning of 'true', which is the concept of truth, which is determined by its use; and that, in turn, is governed by the T-schema. That is why Horwich claims that the impossibility is conceptual. ${ }^{7}$

\section{Minimalism and the Liar}

As we have seen, Horwich's strategy for resolving the Liar consists of restricting the instances of the T-schema that constitute the Minimalist theory of truth so that no such paradox can be formulated. Horwich (1998, p. 42) proposes two conditions that the restriction should meet:

Maximality: Instances of the T-schema cannot be excluded unnecessarily; the Minimal theory of truth should be, if possible, a maximal consistent collection of instances of the T-schema.

Specification: There must be a constructive specification of the instances of the T-schema that are excluded from the Minimalist theory of truth; that specification should be as simple as possible.

As it is well known, McGee (1992) showed that Maximality is not enough to determine which instances of the T-schema should be included in the Minimalist theory. That is because, given any consistent set, $\Delta$, of sentences, there is a maximal consistent set, $\Gamma$, of instances of the T-schema which entails each one of the sentences in $\Delta$. Hence, Maximality does not give us a unique theory of truth. ${ }^{8}$ Horwich has tried to overcome this difficulty by presenting, in his (2010a), a construction which, although not being maximal,

\footnotetext{
${ }^{7}$ See, for example, Horwich (2005, page 102) and Beall and Armour-Garb (2005, pp. 90 ff.) for more details of Horwich's solution of the Liar Paradox.

${ }^{8}$ Moreover, McGee also showed that none of the maximal consistent sets obtained is recursively enumerable and, hence, in this sense of 'constructive', the specification cannot be constructive. In what follows, I will suppose that Horwich's does not expect his theory of truth to be recursively enumerable, which makes perfect sense, since he does not even think that it is a set! (See Horwich 1998, p. 20, fn. 4.) Thanks to Thomas Schindler for prompting this clarification.
} 
offers a constructive specification of the instances of the T-schema we must exclude from the Minimalist theory of truth:

We might say that our language $\mathrm{L}$ is the limit of the expanding sub-languages $L_{0}, L_{1}, L_{2}, \ldots$ where $L_{0}$ lacks the truth predicate; $L_{1}$ (which contains $L_{0}$ ) applies it, via the equivalence schema, to the grounded propositions of $L_{0}$; similarly, $L_{2}$ applies it to the grounded propositions of $L_{1} ; L_{3}$ applies it to the grounded propositions of $\mathrm{L}_{2}$; and so on. Thus an instance of the equivalence schema will be acceptable, even if it governs a proposition concerning truth (e.g. <What John said is true $>$ ), as long as the proposition is grounded. (Horwich 2010a, p. 90)

Horwich seems to describe a construction similar to that proposed in Kripke (1975) and to take the grounded propositions as those whose instances of the T-schema eventually constitute the Minimalist theory of truth. ${ }^{9}$ As I said, Horwich takes propositions as the truth-bearers, which means that, in taking sentences instead of propositions we are not being faithful to Minimalism. Nevertheless, the constructions I am going to discuss cannot be formulated with propositions, which forces me to use sentences. Of course, this means that the constructions presented in this paper need to be understood of as idealized models of the Minimalist theory.

I will follow the formalization of Horwich's construction as presented in Schindler (2015). For perspicuity, let us suppose we have a classical firstorder language, $\mathcal{L}$, the base language, and an expanded language $\mathcal{L}^{+}=$ $\mathcal{L} \cup\{\operatorname{Tr}\}$ with a monadic predicate $\operatorname{Tr}$ intended to represent truth and let us suppose, furthermore, that for every formula $\phi \in \mathcal{L}^{+}$we can express its canonical name $\ulcorner\phi\urcorner$ in $\mathcal{L}$ via some codification. I will suppose that $\mathcal{L}$ is strong enough to prove the Diagonal Lemma, so that the Liar Paradox (and other similar paradoxes) can be formulated.

Given a model for the base language, $\mathcal{N}$, with domain $D$, I will write $\langle\mathcal{N}, A\rangle$ to refer to the model of the expanded language $\mathcal{L}^{+}$whose interpretation of $\operatorname{Tr}$ is $A$, which will be a set of (codes of) sentences of $\mathcal{L}^{+}$. I will use $|\alpha|_{\mathcal{M}}=1$ to mean that the formula $\alpha$ has semantic value 1 in the model $\mathcal{M}$ (and the same for having semantic value 0 ). Given a set of formulas $\Gamma$, I will use $|\Gamma|_{\mathcal{M}}=1$ to mean that, for every $\gamma \in \Gamma,|\gamma|_{\mathcal{M}}=1$.

\footnotetext{
${ }^{9}$ This already raises some doubts concerning whether a deflationist can use the notion of groundedness in order to specify a theory of truth. For the moment, though, let us think of this construction as a mere technicality. I will return to this point later.
} 
Let us begin with the construction. It will consist of a series $H_{\sigma}$ of sets of sentences of $\mathcal{L}^{+}$defined for every ordinal $\sigma$ and relative to a model $\mathcal{N}$ for the base language. We need, first, the following definitions.

\section{Definition}

For any set $A$ of formulas of $\mathcal{L}^{+}, A^{-}=\{\phi: \neg \phi \in A\}$.

For any $\phi \in \mathcal{L}^{+}, T_{\phi}$ is the $\phi$-instance of the T-schema, i.e. $\operatorname{Tr}\ulcorner\phi\urcorner \leftrightarrow \phi$.

For any set $A$ of sentences of $\mathcal{L}^{+}, T_{A}=\left\{T_{\phi}: \phi \in A\right.$ or $\left.\phi \in A^{-}\right\}$.

Horwich's construction is formalized by the following series of sets of sentences of $\mathcal{L}^{+}$, given a model $\mathcal{N}$ for the base language, and for any ordinal $\sigma$ :

$$
\begin{aligned}
H_{0} & =\left\{\phi \in \mathcal{L}:|\phi|_{\mathcal{N}}=1\right\} \\
H_{\sigma+1} & =\left\{\phi \in \mathcal{L}^{+}: H_{\sigma} \cup T_{H_{\sigma}}=\phi\right\} \\
H_{\lambda} & =\bigcup_{\alpha<\lambda} H_{\alpha}
\end{aligned}
$$

where $\lambda$ is a limit ordinal and $\models$ is implication in classical first-order logic.

Horwich claims, in the quote above, that "our language $\mathrm{L}$ is the limit of the expanding sub-languages". He can here be naturally understood as claiming that the formulas at the alleged limit of the sequence are the formulas whose instances of the T-schema will constitute the Minimalist theory of truth. It is also natural to suppose that what Horwich has in mind is a fixed point construction similar to the one presented in Kripke (1975). To wit, we need to find an ordinal $\tau$ where the construction stabilizes; that is, an ordinal $\tau$ such that $H_{\tau}=H_{\tau+1}$.

If we want to show the existence of a fixed point, it is sufficient to prove that the series is monotonic.

Lemma 2.1 (Monotonicity) If $\tau \leq \rho$, then $H_{\tau} \subseteq H_{\rho}$.

Proof It follows immediately from the fact that $\mid=$ is monotonic.

Thus, in each $H_{\sigma}$ you keep the sentences present in the previous elements of the series and, in any case, you add new formulas with the use of a set of instances of the T-schema. As it is well known, monotonicity implies that such construction will eventually reach a fixed point.

Theorem 2.2 (Fixed point) There is an ordinal $\tau$ such that $H_{\tau}=H_{\tau+1}$. 
I will call the fixed point of the construction $H$. Hence, at this point, Horwich's theory of truth, the Minimalist theory of truth, can be characterized as $T_{\boldsymbol{H}}$.

As it stands, though, such a construction does not specify a satisfactory theory of truth. Let us see why. As Schindler (2018) notes, it follows, by compactness and by monotonicity, that Horwich's construction reaches the fixed point at the first limit ordinal; that is, $\boldsymbol{H}=H_{\omega} \cdot{ }^{10}$

This means that $T_{\boldsymbol{H}}$ will not contain any instance of the T-schema of any non trivial truth-theoretic generalization like, for example, 'for any sentence $x, \operatorname{Tr}\ulcorner x \rightarrow x\urcorner$. This is due to the fact that in general, the instances of a universal statement do not imply the universal statement itself. ${ }^{11}$ This fact, as Horwich himself admits, ${ }^{12}$ clearly jeopardizes the explanatory fundamentality of $T_{\boldsymbol{H}}$.

In order to see if a stronger fixed point can be reached, we need to introduce Kripke's fixed point construction (as in Kripke 1975) using the supervaluational scheme. I will use that scheme in order to show that certain variations of the construction just offered produce fixed-points that can be embedded in or are equal to some of its versions.

\section{Kripke and Supervaluations}

With the same notation as above, let us first define the supervaluational scheme, which is a three-valued valuation ||$^{s}$ that will take as semantic values $0,1 / 2$ and 1 . Let $\bar{D}$ be the set of (codes of) sentences of $\mathcal{L}^{+}$. For any $\phi \in \mathcal{L}^{+}$, any model, $\mathcal{N}$, for the base language $\mathcal{L}$ and any set of (codes of) sentences $X,|\psi|_{\langle\mathcal{N}, X\rangle}^{s}$ is defined in the following way:

$$
\begin{aligned}
& |\psi|_{\langle\mathcal{N}, X\rangle}^{S}=1 \text { iff, for every } Y \text {, such that } X \subseteq Y \subseteq \bar{D}-X^{-},|\psi|_{\langle\mathcal{N}, Y\rangle}=1 ; \\
& |\psi|_{\langle\mathcal{N}, X\rangle}^{S}=0 \text { iff, for every } Y \text {, such that } X \subseteq Y \subseteq \bar{D}-X^{-},|\psi|_{\langle\mathcal{N}, Y\rangle}=0 ;
\end{aligned}
$$

\footnotetext{
${ }^{10}$ Given Lemma 2.1 it is enough to show that $H_{\omega+1} \subseteq H_{\omega}$. To see this, suppose that $\phi \in H_{\omega+1}$. In this case, by definition of $H_{\omega+1}, H_{\omega} \cup T_{H_{\omega}} \models \phi$. By compactness of first-order logic, there exists a finite $\Delta \subseteq H_{\omega} \cup T_{H_{\omega}}$ such that $\Delta \models \phi$. Since $\Delta$ is finite, there will be a natural number $n$ such that $\Delta \subseteq H_{n} \cup T_{H_{n}}$. By monotonicity of first-order logic, it follows that $H_{n} \cup T_{H_{n}}=\phi$ and, hence, $\phi \in H_{n+1} \subseteq H_{\omega}$, as desired.

${ }^{11}$ This is a variant of a general problem Horwich has to face. For more details, responses and rejoinders on this issue see, for example, Gupta $(1993 a, b)$, Soames $(1997,1999)$, ArmourGarb (2004, 2010), Raatikainen (2005), Horwich (1998, page 137), Horwich (2010b, pp. 43-45, 92-96) and Oms (2018).

${ }^{12}$ See, for example, Horwich (2010a, p. 96, fn. 16).
} 


$$
|\psi|_{\langle\mathcal{N}, X\rangle}^{s}=1 / 2 \text { otherwise. }
$$

Let us define now the following series of sentences of $\mathcal{L}^{+}$, for any ordinal $\sigma$,

$$
\begin{aligned}
V F_{0} & =\varnothing \\
V F_{\sigma+1} & =\left\{\phi \in \mathcal{L}^{+}:|\phi|_{\left\langle\mathcal{N}, V F_{\sigma}\right\rangle}^{s}=1\right\} \\
V F_{\lambda} & =\bigcup_{\alpha<\lambda} V F_{\alpha}
\end{aligned}
$$

where $\lambda$ is a limit ordinal.

As in the case of the previous section, the construction is monotonic.

Lemma 3.1 (Monotonicity, Kripke 1975) If $\theta \leq \rho$, then $V F_{\theta} \subseteq V F_{\rho}$.

For the same considerations as in Theorem 2.2 there will exist a fixed point of the construction, that is, an ordinal $\rho$ such that $V F_{\rho}=V F_{\rho+1}$. I will call this fixed point $V F$.

Following Kripke (1975) and Field (2008), we can now define variations on the supervaluational scheme by imposing a condition $\Phi$ on the candidate extensions of the truth predicate. These restrictions will create other fixed points that will be supersets of $\boldsymbol{V F}$. In order to proceed, let us define $|\psi|_{\langle\mathcal{N}, X\rangle}^{\Phi, s}$ more generally:

$$
\begin{aligned}
& |\psi|_{\langle\mathcal{N}, X\rangle}^{\Phi, S}=1 \text { iff, for every } Y \text {, such that } \Phi(Y) \text { and } X \subseteq Y \subseteq \bar{D}-X^{-}, \\
& |\psi|_{\langle\mathcal{N}, Y\rangle}=1 ; \\
& |\psi|_{\langle\mathcal{N}, X\rangle}^{\Phi, s}=0 \text { iff, for every } Y \text {, such that } \Phi(Y) \text { and } X \subseteq Y \subseteq \bar{D}-X^{-}, \\
& |\psi|_{\langle\mathcal{N}, Y\rangle}=0 ; \\
& |\psi|_{\langle\mathcal{N}, X\rangle}^{\Phi, S}=1 / 2 \text { otherwise }
\end{aligned}
$$

In this definition, I am presupposing that there will always be a $Y$ that satisfies the condition $\Phi$ and such that $X \subseteq Y \subseteq \bar{D}-X^{-} \cdot{ }^{13}$ Given a condition $\Phi$, I will call $V F_{\sigma}^{\Phi}$ the $\sigma$ stage of the construction using $\Phi$ as the property to be

\footnotetext{
${ }^{13}$ If this were not the case, the construction would have to be adjusted via the addition of a fourth semantic value to represent the situation where there is no appropriate $Y$. Note that, if that fourth semantic value is not defined and there are no $Y^{\prime}$ s satisfying the condition $\Phi$ and such that $X \subseteq Y \subseteq \bar{D}-X^{-}$, then all sentences would trivially have the values 1 and 0 . See Kripke (1975, page 711) and Field (2008, page 178) for more details.
} 
satisfied by the candidate extensions of the truth predicate. I will call $V F^{\Phi}$ the fixed point of such a construction.

We can now consider now the following fixed points corresponding to the following conditions:

The vacuous condition: $\boldsymbol{V F}$

Consistency: $V F^{c}$

Closure under classical deduction: $V \boldsymbol{F}^{c d}$

Maximal consistency: $\boldsymbol{V} \boldsymbol{F}^{m c}$

A trivial generalization of Lemma 3.1 together with the considerations in Theorem 2.2 show that all of $\boldsymbol{V} \boldsymbol{F}, \boldsymbol{V} \boldsymbol{F}^{c}, \boldsymbol{V} \boldsymbol{F}^{c d}$ and $\boldsymbol{V} \boldsymbol{F}^{m c}$ exist. Moreover, as Field (2008, page 180) shows, all of them are consistent.

\section{Constructions}

Recall that the main problem with $\boldsymbol{H}$ was that it did not contain many truththeoretical generalizations, which, if it is to be explanatorily fundamental, it should contain. The reason for this was the fact that, in general, universal statements are not implied by their instantiations. Horwich has tried to overcome this difficulty in a postscript to his (1998) and in Horwich (2010b, pp. 43-45, 92-96), where he defends slightly different strategies that can be interpreted as using some rule akin to the $\omega$-rule. ${ }^{14}$ Schindler (2018) explores what would happen to the construction offered in Section 2 if we added the $\omega$-rule to our logical stock. The fixed point construction can be defined as above with the use of $\vdash_{\omega}$ instead of $\mid=$. Let us call the fixed point we obtain in this new construction S. As Schindler (2018) shows, this fixed point is embedded in Kripke's fixed point using the supervaluational scheme with the vacuous condition on the candidate extensions of the truth predicate:

Proposition $4.1 S \subseteq V F^{15}$

As we are going to see, though, $S$ is still too weak. Hence, it is worth trying to obtain some other version of the construction that allows us to arrive at stronger theories of truth. Let us see if this can be done.

\footnotetext{
${ }^{14}$ For some criticisms to the use of that rule see Raatikainen (2005).

${ }^{15}$ Question: is the inclusion proper?
} 
As we saw in the construction of the fixed point $\boldsymbol{H}$, the base level $H_{0}$ contained all the true sentences of the base language $\mathcal{L}$ that have semantic value 1 in the base model. Hence, $H_{0}$ can be seen as a theory of all the nonsemantic facts given by the ground model $\mathcal{N}$. Consequently, according to Horwich, $H_{0}$ together with the corresponding theory of truth should explain all our uses of truth and should determine all that can be known about truth at all. In a sense then, $H_{0}$ must be fixed throughout the construction. Right now it is fixed in the sense that, by monotonicity, it remains in every $H_{\sigma}$ 's. However, we can strengthen the sense in which $H_{0}$ is fixed in the construction by treating the expressions in the base language $\mathcal{L}$, given the base model $\mathcal{N}$, as logical constants and accordingly define a new relation of logical consequence, $\left.\right|_{\mathcal{N}}:\left.\Gamma\right|_{\mathcal{N}} \phi$ iff, for all $X$ such that $|\Gamma|_{\langle\mathcal{N}, X\rangle}=$ $1,|\phi|_{\langle\mathcal{N}, X\rangle}=1$. So, in this case, the base model would remain fixed and only the extension of $\operatorname{Tr}$ would vary from model to model. Hence, for any sentence $\phi$ of the base language $\mathcal{L},|\phi|_{\mathcal{N}}=1$ iff, $\left.\right|_{\mathcal{N}} \phi$. Clearly, $\Gamma \models \phi$ implies $\Gamma \mid=_{\mathcal{N}} \phi$.

To my mind, this restriction on the relation of logical consequence squares well with Minimalism and it is not an ad hoc move. As I have said, the Minimalist theory of truth cannot be expected to explain all our uses of truth in, say, the domain of biology; in order to explain all our uses of truth in biology we will need the Minimalist theory of truth together with the appropriate principles of biology. ${ }^{16}$ So, suppose we wanted to explore which facts regarding truth follow in biology from the Minimalist theory. What we would do is to keep the biological facts fixed and use the Minimalist theory of truth to explore what follows. It seems to me that the sense in which we would thus fix the biological facts would be akin to the sense I am proposing here in which we fix $H_{0}$ (which, recall, is taken to represent the theory of all non-semantic facts). This is because we do not contemplate the possibility that biology could have been different when we ask ourselves what follows from the Minimalist theory of truth in biology. In the same manner, we do not contemplate the possibility that $H_{0}$ could have been different when we ask ourselves what follows from the Minimalist theory of truth and $H_{0}$; that is to say, we do not contemplate models other than those that make all sentences in $H_{0}$ true.

Accordingly, we can now define the construction again as follows:

\footnotetext{
${ }^{16}$ What is important is that such principles do not use the truth predicate, for if they did, they would show that we need to go beyond the instances of the T-schema in order to explain all facts concerning truth.
} 


$$
\begin{aligned}
H_{0}^{*} & =\left\{\phi \in \mathcal{L}:|\phi|_{\mathcal{N}}=1\right\} \\
H_{\sigma+1}^{*} & =\left\{\phi \in \mathcal{L}^{+}: H_{\sigma}^{*} \cup T_{H_{\sigma}^{*}}=_{\mathcal{N}} \phi\right\} \\
H_{\lambda}^{*} & =\bigcup_{\alpha<\lambda} H_{\alpha}^{*}
\end{aligned}
$$

where $\lambda$ is a limit ordinal and $\models_{\mathcal{N}}$ is the new restricted logical consequence relation relative to the base model $\mathcal{N}$.

As before, the construction is monotonic and there exists a fixed point, let us call it $\boldsymbol{H}^{*}$. Now we can show that $\boldsymbol{H}^{*}$ is just $\boldsymbol{V F} .{ }^{17}$

Lemma 4.2 For any ordinal $\sigma, H_{\sigma}^{*} \subseteq V F_{\sigma+1}$.

Proof The proof proceeds by induction on $\sigma$. For the base case, suppose $\sigma=0$, then if $\phi \in H_{0}^{*}$, by the definition of $H_{0}^{*}$ we have that $\phi \in \mathcal{L}$ and $=_{\mathcal{N}} \phi$, so that $|\phi|_{\mathcal{N}}=1$. This means that for every $Y$ such that $V F_{0} \subseteq Y \subseteq \bar{D}-V F_{0}^{-}$, $|\phi|_{\langle\mathcal{N}, Y\rangle}=1$ (just because the value of $\phi$, being from the base language, is independent of the choice of the extension of the truth predicate) and hence, $\phi \in V F_{1}$.

For the successor case, suppose that $H_{\sigma}^{*} \subseteq V F_{\sigma+1}$. We need to show that $H_{\sigma+1}^{*} \subseteq V F_{\sigma+2}$. Take $\phi \in H_{\sigma+1}^{*}$. This means that $H_{\sigma}^{*} \cup T_{H_{\sigma}^{*}} \models_{\mathcal{N}} \phi$. Now what we need to show is that $|\phi|_{\left\langle\mathcal{N}, V F_{\sigma+1}\right\rangle}^{s}=1$, that is, we need to show that for any $Y$ such that $V F_{\sigma+1} \subseteq Y \subseteq \bar{D}-V F_{\sigma+1}^{-},|\phi|_{\langle\mathcal{N}, Y\rangle}=1$. Take any $Y$ satisfying the conditions above. We will next show that $\left|H_{\sigma}^{*}\right|_{\langle\mathcal{N}, Y\rangle}=$ 1 and that $\left|T_{H_{\sigma}^{*}}\right|_{\langle\mathcal{N}, Y\rangle}=1$. This means that, since we are supposing that $H_{\sigma}^{*} \cup T_{H_{\sigma}^{*}} \models_{\mathcal{N}} \phi,|\phi|_{\langle\mathcal{N}, Y\rangle}=1$, which is what we want to prove.

First, let us show that $\left|H_{\sigma}^{*}\right|_{\langle\mathcal{N}, Y\rangle}=1$. Take $\phi \in H_{\sigma}^{*}$. By the induction hypothesis, $\phi \in V F_{\sigma+1}$ and, by monotonicity (Lemma 3.1), $\phi \in V F_{\sigma+2}$, which means that $|\phi|_{\left\langle\mathcal{N}, V F_{\sigma+1}\right\rangle}^{S}=1$ and hence, for any $Y^{\prime}$ such that $V F_{\sigma+1} \subseteq Y^{\prime} \subseteq$ $\bar{D}-V F_{\sigma+1}^{-},|\phi|_{\left\langle\mathcal{N}, Y^{\prime}\right\rangle}=1$. So, in particular, $|\phi|_{\langle\mathcal{N}, Y\rangle}=1$.

Second, let us show that $\left|T_{H_{\sigma}^{*}}\right|_{\langle\mathcal{N}, Y\rangle}=1$. Again, take $\phi \in H_{\sigma}^{*}$. We just showed that $|\phi|_{\langle\mathcal{N}, Y\rangle}=1$. Since, by the induction hypothesis and the construction of $Y, H_{\sigma}^{*} \subseteq V F_{\sigma+1} \subseteq Y, \phi \in Y$ and, hence, $|\operatorname{Tr}\langle\phi\rangle|_{\langle\mathcal{N}, Y\rangle}=1$. Consequently, $|\operatorname{Tr}\ulcorner\phi\urcorner \leftrightarrow \phi|_{\langle\mathcal{N}, Y\rangle}=1$.

Now take $\phi \in H_{\sigma}^{*-}$. Then, $\neg \phi \in H_{\sigma}^{*},|\neg \phi|_{\langle\mathcal{N}, Y\rangle}=1$ and, hence, $|\phi|_{\langle\mathcal{N}, Y\rangle}=$ 0 . Meanwhile, by the induction hypothesis, $\neg \phi \in V F_{\sigma+1}$ and, therefore, $\phi \in$ $V F_{\sigma+1}^{-}$. But, since $Y \subseteq \bar{D}-V F_{\sigma+1}^{-}, \phi \notin Y$ and consequently, $|\operatorname{Tr}\langle\phi\rangle|_{\langle\mathcal{N}, Y\rangle}=0$. Therefore, $|\operatorname{Tr}\ulcorner\phi\urcorner \leftrightarrow \phi|_{\langle\mathcal{N}, Y\rangle}=1$.

\footnotetext{
${ }^{17}$ Thanks to Elia Zardini for suggesting this line of thought.
} 
For the Limit case, suppose that for all ordinals $\sigma, \sigma<\lambda$, for a given limit ordinal $\lambda, H_{\sigma}^{*} \subseteq V F_{\sigma+1}$. We need to show that $H_{\lambda}^{*} \subseteq V F_{\lambda+1}$. Take any $\phi \in H_{\lambda}^{*}$. By the definition of $H_{\lambda}^{*}$, for some $\rho, \rho<\lambda, \phi \in H_{\rho}^{*}$. By the induction hypothesis it follows that $\phi \in V F_{\rho+1}$ and hence, by the definition of $V F_{\lambda}$, $\phi \in V F_{\lambda}$. This, by monotonicity (Lemma 3.1), implies that $\phi \in V F_{\lambda+1}$.

Lemma 4.3 For any ordinal $\sigma, V F_{\sigma} \subseteq H_{\sigma}^{*}$.

Proof The proof proceeds by induction on $\sigma$. The base case is clear from the definition of $V F_{0}$.

For the successor case, suppose that $V F_{\sigma} \subseteq H_{\sigma}^{*}$. We need to show that $V F_{\sigma+1} \subseteq H_{\sigma+1}^{*}$. Take $\phi \in V F_{\sigma+1}$. This means that $|\phi|_{\left\langle\mathcal{N}, V F_{\sigma}\right\rangle}^{s}=1$, that is, for any $Y$ such that $V F_{\sigma} \subseteq Y \subseteq \bar{D}-V F_{\sigma}^{-},|\phi|_{\langle\mathcal{N}, Y\rangle}=1$. We need to show that $H_{\sigma}^{*} \cup T_{H_{\sigma}^{*}}=_{\mathcal{N}} \phi$. So take any model $\langle\mathcal{N}, X\rangle$ (where $\mathcal{N}$ is the base model used in the construction which, recall, is now fixed) such that $\left|H_{\sigma}^{*} \cup T_{H_{\sigma}^{*}}\right|\langle\mathcal{N}, X\rangle=1$. We need to show that $|\phi|_{\langle\mathcal{N}, X\rangle}=1$. In order to do that it will be enough to show that $V F_{\sigma} \subseteq X \subseteq \bar{D}-V F_{\sigma}^{-}$.

First, let us show that $V F_{\sigma} \subseteq X$. Take $\psi \in V F_{\sigma}$. Then, by the induction hypothesis, $\psi \in H_{\sigma}^{*}$, which means that, by supposition, $|\psi|_{\langle\mathcal{N}, X\rangle}=1$. Moreover, if $\psi \in H_{\sigma}^{*}$, then $\operatorname{Tr}\ulcorner\psi\urcorner \leftrightarrow \psi \in T_{H_{\sigma}^{*}}$ and, again by supposition, $|\operatorname{Tr}\ulcorner\psi\urcorner \leftrightarrow \psi|_{\langle\mathcal{N}, X\rangle}=1$. Therefore $|\operatorname{Tr}\ulcorner\psi\urcorner|_{\langle\mathcal{N}, X\rangle}=1$, which implies that $\psi \in X$

Second, let us show that $X \subseteq \bar{D}-V F_{\sigma}^{-}$. Take $\psi \in X$. We want to show that $\psi \notin V F_{\sigma}^{-}$. Suppose, for the sake of contradiction, that $\psi \in V F_{\sigma}^{-}$. In that case, $\neg \psi \in V F_{\sigma}$ and, by the induction hypothesis, $\neg \psi \in H_{\sigma}^{*}$ and, hence, $\psi \in H_{\sigma}^{*-}$. Therefore, by the definition of $T_{H_{\sigma}^{*}}, \operatorname{Tr}\ulcorner\psi\urcorner \leftrightarrow \psi \in T_{H_{\sigma}^{*}}$ and hence, by supposition, $|\operatorname{Tr}\ulcorner\psi\urcorner \leftrightarrow \psi|_{\langle\mathcal{N}, X\rangle}=1$. But, if $\neg \psi \in H_{\sigma}^{*}$ then, again by supposition, $|\neg \psi|_{\langle\mathcal{N}, X\rangle}=1$ and, hence, $|\psi|_{\langle\mathcal{N}, X\rangle}=0$. Consequently $|\operatorname{Tr}\ulcorner\psi\urcorner|_{\langle\mathcal{N}, X\rangle}=0$, which means that $\psi \notin X$. Contradiction.

For the limit case, suppose that for all ordinals $\sigma, \sigma<\lambda(\lambda$ a limit ordinal), $V F_{\sigma} \subseteq H_{\sigma}^{*}$. We need to show that $V F_{\lambda} \subseteq H_{\lambda}^{*}$. Take any $\phi \in V F_{\lambda}$. By the definition of $V F_{\lambda}$, for some $\rho, \rho<\lambda, \phi \in V F_{\rho}$. By the induction hypothesis it follows that $\phi \in H_{\rho}^{*}$ and hence, by the definition of $H_{\lambda}^{*}, \phi \in H_{\lambda}^{*}$.

\section{Corollary $4.4 H^{*}=V F$}

Note also that, as the following remark shows, all the axioms of Minimalism are grounded, that is, they are in $H^{*}$.

Remark 4.5 $T_{\boldsymbol{H}^{*}} \subseteq \boldsymbol{H}^{*}$ 
Proof Take any $\phi \leftrightarrow \operatorname{Tr}\ulcorner\phi\urcorner \in T_{\boldsymbol{H}^{*}}$. Clearly, $\boldsymbol{H}^{*} \cup T_{\boldsymbol{H}^{*}} \equiv_{\mathcal{N}} \phi \leftrightarrow \operatorname{Tr}\ulcorner\phi\urcorner$. Hence, by the fact that $\boldsymbol{H}^{*}$ is a fixed point, it follows that $\phi \leftrightarrow \operatorname{Tr}\ulcorner\phi\urcorner \in \boldsymbol{H}^{*}$.

\section{The Minimalist Theory of Truth}

We should now ask ourselves whether the theory of truth Horwich is proposing is satisfactory. Recall that we can now characterize what is, according to our reading of Horwich (2010a), the Minimalist theory of truth (the appropriate collection of instances of the T-schema) in a precise way: $T_{\boldsymbol{H}^{*}}$. Unfortunately, $T_{\boldsymbol{H}^{*}}$ will not yet constitute a good candidate for the Minimalist theory of truth. Let us see why.

It is well known that $V F$ has many unsatisfactory properties. Specifically, the following statements are not included in it:

1. For any sentence $x$; $\operatorname{Tr}\ulcorner\neg \neg x\urcorner$ if, and only if, $\operatorname{Tr}\ulcorner x\urcorner$.

2. For any sentences $x, y$; $\operatorname{Tr}\ulcorner x \vee y\urcorner$ if, and only if, $\operatorname{Tr}\ulcorner x\urcorner$ or $\operatorname{Tr}\ulcorner y\urcorner$.

3. For any sentences $x, y$; $\operatorname{Tr}\ulcorner x \wedge y\urcorner$ if, and only if, $\operatorname{Tr}\ulcorner x\urcorner$ and $\operatorname{Tr}\ulcorner y\urcorner$.

4. For any sentences $x, y$; if $\operatorname{Tr}\ulcorner x \rightarrow y\urcorner$ and $\operatorname{Tr}\ulcorner x\urcorner$, then $\operatorname{Tr}\ulcorner y\urcorner$.

The problem is that we can find some instances of these principles that are not in $\boldsymbol{V F}$. Hence, they will neither be in $\boldsymbol{H}^{*}$. As we are going to see, this makes the construction above unsuitable as an appropriate Minimalist theory of truth.

Let us see exactly what it means to say that laws $1-4$ are not in $\boldsymbol{H}^{*}$. We have to ask ourselves, first, how we should understand what $\boldsymbol{H}^{*}$ is. The way the construction is devised makes it natural to consider the set $\boldsymbol{H}^{*} \cup \boldsymbol{H}^{*-}$ as the set of grounded sentences; specifically, $\boldsymbol{H}^{*}$ is the set of determinately true sentences (that is, supposing there are not vague predicates nor other sources of indeterminacy in Horwich's epistemic sense, the sentences which it is conceptually possible to know) and $\boldsymbol{H}^{*-}$ is the set of determinately false sentences (that is, those sentences whose negations are determinately true). To continue with this picture, notice that, as we saw, it is natural to interpret $H_{0}^{*}$ as the theory of all non-semantic facts given by the ground model $\mathcal{N}$ and, accordingly, we can take $\boldsymbol{T}=\left\{\phi: T_{\boldsymbol{H}^{*}} \cup H_{0}^{*}=\phi\right\}$ as representing everything that can be known about truth at all. So, it is natural to expect the following proposition. 


\section{Proposition $5.1 T \subseteq H^{*}$}

Proof Suppose $\phi \notin \boldsymbol{H}^{*}$. Then, for no $\rho, H_{\rho}^{*} \cup T_{H_{\rho}^{*}}=_{\mathcal{N}} \phi$ and, in particular, $\boldsymbol{H}^{*} \cup T_{\boldsymbol{H}^{*}} \not \models_{\mathcal{N}} \phi$. Since $H_{0}^{*} \subseteq \boldsymbol{H}^{*}$, we conclude that $H_{0}^{*} \cup T_{\boldsymbol{H}^{*}} \nvdash_{\mathcal{N}} \phi$. Consequently, $H_{0}^{*} \cup T_{\boldsymbol{H}^{*}} \not \models \phi$ and hence, $\phi \notin T{ }^{18}$

Thus, $\boldsymbol{H}^{*}$ contains everything we can know about truth at all. So the fact that principles like 1-4 are not in $\boldsymbol{H}^{*}$ is a major problem for Minimalism and it clearly jeopardizes its explanatory power.

Can this situation be ameliorated? I think so. It is well known that other fixed points of the supervaluational scheme, such as those I presented above-defined using restrictions on the candidate extensions of the truth predicate-are much better behaved with respect to principles like 1-4. So, the question is whether Horwich's construction can be manipulated in such a way that the fixed point we arrive at in the end is stronger than $V F$; ideally $V \boldsymbol{F}^{m c}$. That manipulation should be motivated, however, by independent reasons beyond the fact that $\boldsymbol{V F}$ does not contain principles 1-4. This is the case, if we bear in mind Horwich's position with respect to the Liar Paradox.

Let us begin by asking ourselves which model of the expanded language is the actual model; which model captures the actual world? First, since Horwich's position regarding the Liar defends, as we have seen in Section 1 , that any sentence (in particular the Liar) is such that it is true or it is false, then the extension of the truth predicate in the actual model will have to be, at least, complete: there will be no undecided cases of being true.

On the other hand, Horwich adopts classical logic, which means that not only are all sentences either true or false, but also that it is not the case that any of them are true and false. Hence, it seems natural to expect the extension of the truth predicate to be consistent; that is, no sentence is both true and false. All this means that the extension of the truth predicate in the actual model should be maximally consistent. ${ }^{19}$ As far as I can see, this

\footnotetext{
${ }^{18}$ Alternatively, we could define the set representing all we can know about truth with the restricted notion of logical consequence, $\boldsymbol{T}_{\mathcal{N}}=\left\{\phi: T_{\boldsymbol{H}^{*}} \cup H_{0}^{*} \models_{\mathcal{N}} \phi\right\}$. In this case, $\boldsymbol{T}_{\mathcal{N}}=\boldsymbol{H}^{*}$. That $\boldsymbol{T}_{\mathcal{N}} \subseteq \boldsymbol{H}^{*}$ is proved as in Proposition 5.1. That $\boldsymbol{H}^{*} \subseteq \boldsymbol{T}_{\mathcal{N}}$ is proved by showing that for every ordinal $\sigma, T_{\boldsymbol{H}^{*}} \cup H_{0}^{*}=H_{\sigma}^{*}$. The proof proceeds by induction on $\sigma$. The base case and the limit case are clear; as for the successor case, suppose that $T_{\boldsymbol{H}^{*}} \cup H_{0}^{*}=H_{\sigma}^{*}$. Take $\phi \in H_{\sigma+1}^{*}$. By the definition of $H_{\sigma+1}^{*}, H_{\sigma}^{*} \cup T_{H_{\sigma}^{*}}=\mathcal{N} \phi$. Now, from the induction hypothesis and the fact that $T_{H_{\sigma}^{*}} \subseteq T_{\boldsymbol{H}^{*}}$, it follows that $T_{\boldsymbol{H}^{*}} \cup H_{0}^{*} \models \mathcal{N} H_{\sigma}^{*} \cup T_{H_{\sigma}^{*}}$. Hence, $T_{\boldsymbol{H}^{*}} \cup H_{0}^{*}=\phi$. For the purposes in this paper, I do not need to decide between $T$ and $T_{\mathcal{N}}$ because what I need is the result of Proposition 5.1, which holds in both cases.

${ }^{19}$ This is not to say that $\boldsymbol{H}^{*}$ must be a maximally consistent set (which is not); $\boldsymbol{H}^{*}$ represents, as we have seen, the set of determinately true sentences (in Horwich's epistemic
} 
makes perfect sense for, if we think of which is the set of true sentences in the model that represents the actual world as the theory of that model, it is reasonable to take that set as being maximally consistent. ${ }^{20}$

These considerations naturally suggest that we must restrict our attention, in general, to models whose extension of the truth predicate is maximally consistent. Hence, we can bring this restriction to the consequence relation used in the construction.

Following this line of thought I will call a model $\mathcal{M}$ for $\mathcal{L}^{+}$mc-acceptable if its interpretation of $\operatorname{Tr}$ is consistent and, for every $\phi \in \mathcal{L}^{+}$, either $\ulcorner\phi\urcorner$ or $\ulcorner\neg \phi\urcorner$ (but not both) belong to this interpretation. We can now restrict further our already restricted logical consequence relation $\models_{\mathcal{N}}$ to mc-acceptable models. Let us refer to this new relation as $\models_{\mathcal{N}}^{m c}$.

The definition of the new series will be the same, but replacing $\models_{\mathcal{N}}$ by $\models_{\mathcal{N}}^{m c}$. Lemma 2.1 can be proved in the same way (essentially it uses the fact that, if $\phi \in A$, for any sentence $\phi$ and set of sentences $A$, then $A \models_{\mathcal{N}}^{m c} \phi$ ) and hence, a fixed point of the construction, let us call it $\boldsymbol{H}^{m c}$, will exist. Lemmas 4.2 and 4.3 can easily be proved for $\boldsymbol{H}^{m c}$ and $\boldsymbol{V} \boldsymbol{F}^{m c}$ to show that $\boldsymbol{H}^{m c}=\boldsymbol{V} \boldsymbol{F}^{m c}$

Now all the instances of laws 1-4 are validated in $\boldsymbol{H}^{m c}$ and we have found some independent reasons-Horwich's stance with respect to the Liar-to motivate the adoption of this stronger fixed point. ${ }^{21}$

sense) and what we are considering now is the set of true sentences simpliciter. Moreover, note that any model $\mathcal{M}$ for $\mathcal{L}^{+}$with an extension of the truth predicate, $\operatorname{Tr} \mathcal{M}$, such that $\boldsymbol{H}^{*} \subseteq \operatorname{Tr} \mathcal{M} \subseteq \bar{D}-\boldsymbol{H}^{*-}$ satisfies $T_{\boldsymbol{H}^{*}}$-which is the Minimalist theory of truth-and $\boldsymbol{H}^{*}-$ which contains all that can be known about truth. (This follows immediately from the fact that $\boldsymbol{H}^{*}=V \boldsymbol{F}$.) Then, by the factivity of knowledge, it is reasonable to expect the extension of the truth predicate in the actual model to be a maximally consistent superset of $\boldsymbol{H}^{*}$. Thanks to a anonymous referee for prompting this clarification.

${ }^{20}$ Thanks to José Martínez for suggesting this.

${ }^{21}$ Not everything is good news, though; as Field (2008, pp. $184 \mathrm{ff}$.) shows, some reasonable principles involving the quantifiers (e.g. $\forall n \operatorname{Tr}\ulcorner\phi(n)\urcorner \rightarrow \operatorname{Tr}\ulcorner\forall n \phi(n)\urcorner)$ will fail to hold even in $V F^{m c}$. So the fixed point is not strong enough. Thanks to Lucas Rosenblatt for suggesting that point. Moreover, it is worth noticing that, as Schindler (2018) emphazises, some of the laws that hold in the theory based on maximally consistent extensions of the truth predicate do not hold if one moves to its classical close-off. This is a problem for Horwich's demand that Minimalism be a classical theory of truth. Thanks to an anonymous referee for pressing that point. 


\section{Concluding Remarks}

It is time now to take stock. We have seen in Section 2 that Horwich (1998) proposes two conditions that the restriction of the T-schema should satisfy, the Maximality and the Specification constraints. We have already seen that the former alone is not enough to specify a particular maximal consistent set of instances of the T-schema. We have also seen, in previous sections, Horwich's proposal with respect to the latter. However, neither condition is yet met, even in the case where we take $\boldsymbol{H}^{m c}$ as the set of determinately true sentences. To see why, consider the Truth Teller: the sentence $\tau$ such that $\tau \leftrightarrow \operatorname{Tr}\ulcorner\tau\urcorner$. Notice that $\tau \notin \boldsymbol{H}^{m c}$ and, hence, although $\tau \leftrightarrow \operatorname{Tr}\ulcorner\tau\urcorner \in$ $T$-as the Diagonal Lemma shows that it is a theorem-and, consequently, we can know that the $\tau$-instance of the T-schema is true, we cannot know whether $\tau$ itself is true or not. Notice now that it would be perfectly safe to introduce $\tau$ directly into $H_{0}^{m c}$ (that is, the first stage of the construction of $\left.\boldsymbol{H}^{m c}\right)$. In this case, $\tau$ would obviously be at the fixed point that results from the construction which would be a superset of $\boldsymbol{H}^{m c}$. As Kripke (1975) showed, many maximal incompatible fixed points will extend $\boldsymbol{V} \boldsymbol{F}$ and we will not have any means of deciding which one to adopt as the root of our truth theory.

In any case, suppose that we succeeded in singling out one unique fixed point containing sentences like $\tau$. In that case, we would be able to know $\tau$, because, since it is in $H_{0}^{m c}$, it is also in $T$, which, as we have seen, contains everything we can know about truth. But a mere desire for Maximality seems a poor reason to acquire knowledge about $\tau$ or similar sentences; that is, the Maximality constrain would give us knowledge about many sentences in a way that seems far too arbitrary. This is a consequence of the fact that choosing any maximal fixed point over another will be arbitrary and the fact that we understand the fixed point as capturing all that can be known about truth. Taking all these considerations into account, it seems more reasonable to abandon Maximality and retain only the Specification constrain.

In the previous few sections, I focused on a variation of the constructive specification Horwich proposes. The result of that turned out to be identical to the minimal fixed point of Kripke's construction using the supervaluationist schema without restrictions on the candidate extensions of the truth predicate. Since the result of this construction was too weak, I presented a way to strengthen it in order to obtain the fixed point of Kripke's construction using the supervaluationist schema; but now, with the condition of maximal consistency for the candidate extensions of the truth predicate. 
Next, the following question emerges: given that Kripke's construction already gives us the fixed point, why do we need Horwich's construction?

Horwich (2010a) presents his construction as one that "squares with Minimalism" (Horwich 2010a, p. 92, fn. 12) in the sense that it does not use compositional principles for truth, which are seen as incompatible with Minimalism. This is so because Minimalism understands truth via the Tschema and not via compositional principles à la Tarski. Indeed, Horwich rejects Kripke's construction based on the strong Kleene scheme because it "invokes Tarski-style compositional principles" (Horwich 2010a, p. 92, fn. 12). At this point, then, it is natural to expect that Horwich would also reject the supervaluationist version of Kripke's construction since, although the supervaluational scheme assigns the semantic value on the grounds of ways of making the truth predicate precise, in each of those ways, the semantic value of the sentences is achieved by compositional rules. ${ }^{22} \mathrm{Im}$ portantly, this discussion clearly suggests that Horwich does not take the construction to be a mere technical device to determine which instances of the T-schema are included in the Minimalist theory and that, therefore, it needs to be restricted by strict deflationist constraints-after all, if it were a mere technical device, it would not matter whether it used compositional principles for truth.

However, Horwich's construction relies heavily on the notion of groundedness; he himself claims that "a good solution to the liar paradox should articulate 'grounding' constraints [...] on which particular instances of [the T-schema] are axioms" (Horwich 2010a, page 91). Given that, as I claimed, the construction is not a mere technical device and has to satisfy strict deflationist constraints, using the notion of groundedness might seem to be at odds with Horwich's deflationist view of truth. After all, depending on how we understand the notion of groundedness, if it is constitutive of the notion of truth, it is no longer the case that "our commitment to [the T-schema] accounts for everything we do with the truth predicate" (Horwich 1998, page 121) and hence, it is no longer the case that the T-schema implicitly defines it.

Horwich may have a way out of this situation; he might understand the construction of $\boldsymbol{H}^{m c}\left(\boldsymbol{H}, \boldsymbol{S}, \boldsymbol{H}^{*}\right)$ as a model of how truth claims are explained by some things in the world being in a certain way. ${ }^{23}$ Let me elaborate

\footnotetext{
${ }^{22}$ It could be said that the construction for $\boldsymbol{H}^{*}$ implicitly uses compositional principles for truth in the relation of logical consequence and that, in consequence, Horwich should reject it too. This is not the case, though, as Horwich thinks that truth does not play any role in the foundations of logic (see Horwich 1998, pp. 74-6).

${ }^{23}$ Thanks to Manuel García-Carpintero and Sven Rosenkranz for their helpful insights on
} 
on this. Horwich admits that the correspondence intuition can be accommodated within Minimalism. We can loosely characterize correspondence theories of truth as defending that being true consists of corresponding to facts. Although, obviously, Horwich does not endorse this characterization, he claims:

[W] might hope to accommodate much of what the correspondence theorist wishes to say without retreating an inch from our deflationary position. [...]

It is indeed undeniable that whenever a proposition or an utterance is true, it is true because something in the world is a certain way-something typically external to the proposition or the utterance. For example,[...]

$<$ Snow is white $>$ is true because snow is white.

(Horwich 1998, page 104)

Thus, claims Horwich, the fact that snow is white is explanatorily prior to the fact that $<$ Snow is white $>$ is true; in the same way as the basic laws of nature and the initial state of the universe are explanatorily prior to the fact that snow is white.

I think that it is reasonable to understand the constructions I have discussed in this paper as epistemic models of the relation of explanatory dependence between truth ascriptions and the non-semantic facts. According to this epistemic reading of groundedness, the grounded sentences, those in the fixed point, are those that can be explained-and, hence, those that can be known-given how the world is and given the appropriate instances of the T-schema. ${ }^{24}$

\section{References}

Armour-Garb, Bradley (2004). "Minimalism, the Generalization Problem and the Liar". In: Synthese 139.3, pages 491-512.

this issue.

${ }^{24}$ Thanks to José Martínez, Thomas Schindler, Elia Zardini and two anonymous referees for all their very stimulating comments and suggestions. I am also grateful to the audience at the LOGOS Seminar, especially to Manuel García-Carpintero, Lucas Rosenblatt and Sven Rosenkranz. During the writing of the paper, I have benefitted from the project FFI201570707P of the Spanish Ministry of Economy, Industry and Competitiveness on Localism and Globalism in Logic and Semantics. 
Armour-Garb, Bradley (2010). "Horwichian Minimalism and the Generalization Problem". In: Analysis 70.4, pages 693-703.

Beall, J.C. and Bradley Armour-Garb (2005). "Minimalism, Epistemicism and Paradox". In: Deflationism and Paradox. Edited by J. C. Beall and Bradley Armour-Garb. Oxford: Oxford University Press. Chapter 6.

Field, Hartry (2008). Saving Truth From Paradox. Oxford: Oxford University Press.

Gupta, Anil (1993a). “A Critique of Deflationism". In: Philosophical Topics 21.2, pages $57-81$.

- (1993b). "Minimalism". In: Philosophical Perspectives 7, pages 359-69.

Horwich, Paul (1997). "The Nature of Vagueness". In: Philosophy and Phenomenological Research 57, pages 929-36.

- (1998). Truth. 2nd. Oxford: Oxford University Press.

- (2001). "A Defense of Minimalism". In: Synthese 126.1-2, pages 149-65. Reprinted in Paul Horwich (2010b). Truth-Meaning-Reality. Oxford: Oxford University Press, pages 35-56.

- (2005). Reflections on Meaning. Oxford: Oxford University Press.

- (2010a). "A Minimalist Critique of Tarski". In: Truth-Meaning-Reality. Oxford: Oxford University Press, pages 79-97.

- (2010b). Truth-Meaning-Reality. Oxford: Oxford University Press.

Kripke, Saul A. (1975). "Outline of a Theory of Truth". In: Journal of Philosophy 72.19, pages 690-716.

McGee, Vann (1992). "Maximal Consistent Sets of Instances of Tarski's Schema (T)". In: Journal of Philosophical Logic 21.3, pages 235-241.

Oms, Sergi (2018). "Conceivability, Minimalism and the Generalization Problem". In: Dialogue.

Raatikainen, Panu (2005). “On Horwich's Way Out". In: Analysis 65.287, pages $175-177$.

Restall, Greg (2005). "Minimalists about Truth Can (and Should) Be Epistemicists, and it Helps if They Are Revision Theorists too". In: Deflationism and Paradox. Edited by J.C. Beall and Bradley Armour-Garb. Oxford: Oxford University Press. Chapter 7.

Schindler, Thomas (2015). "A Disquotational Theory of Truth as Strong as $Z_{2}^{-\prime \prime}$. In: Journal of Philosophical Logic 44, pages 395-410.

- (2018). "A Note on Horwich's Notion of Grounding". In: Synthese.

Soames, Scott (1997). "The Truth About Deflationism". In: Philosophical Issues, 8, Truth. Edited by Enrique Villanueva. Atascadero, California: Ridgeview Publishing Company, pages 1-44.

- (1999). Understanding Truth. Oxford: Oxford University Press. 